\title{
Endovascular treatment of thoracic central vein pathology caused by pacemaker leads
}

\author{
Radosław Pietura ${ }^{1}$, Tomasz Smyk ${ }^{2}$, Łukasz Tułecki ${ }^{3}$, Andrzej Kleinrok² ${ }^{2}$ Michał Toborek ${ }^{1}$, Andrzej Kutarski ${ }^{4}$
}

'Department of Radiography, Medical University of Lublin, Lublin, Poland

2Department of Cardiology, Pope John Paul II Regional Hospital, Zamosc, Poland

${ }^{3}$ Department of Cardiac Surgery, Pope John Paul II Regional Hospital, Zamosc, Poland

${ }^{4}$ Department of Cardiology, Medical University of Lublin, Lublin, Poland

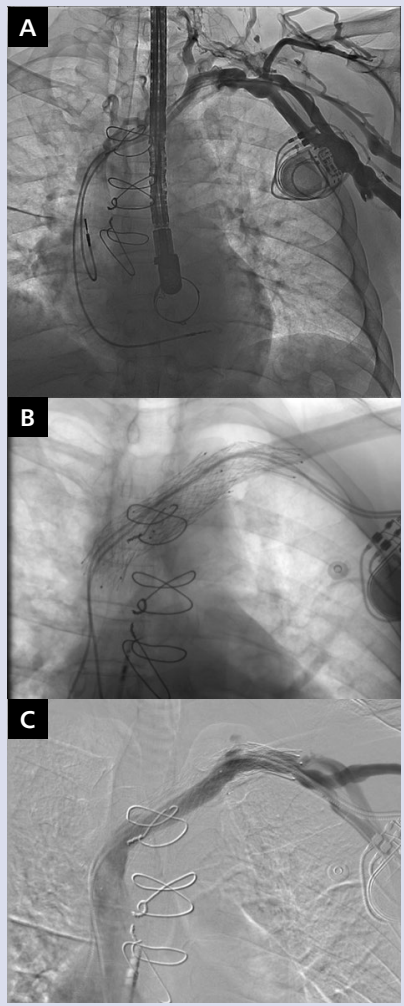

Figure 1. A. Critical stenosis of the left brachiocephalic (BCV) vein and collaterals; $\mathbf{B}$. Stents in the BCV after DDD pacemaker reimplantation; C. 12-month follow-up phlebography

\section{References}

1. ŻebrowskiMR, KruckaA,BednarkiewiczZ, et al. [Complications of permanent implantation of the cardiac stimulaimplantation of the cardiac stimula-
tion system. Part II. Thromboembolic complications]. Forum Kardiologów. 2004: 9: 31-34.

Lickfett $\mathrm{L}$ Bitzen $\mathrm{A}$ Arepally $\mathrm{A}$ et al. Incidence of venous, Incidence of venous obstruction following insertion of an implantable cardioverter defibrillator. A study of systematic contrast venography on patients presenting for their first elective ICD generator replacement. Europace. 2004; 6(1): 25-31, indexed in Pubmed: 1469772

Antonelli D, Turgeman Y, Kaveh Z, et al. Short-term thrombosis after transvenous permanent pacemaker insertion. Pacing Clin Electrophysiol. 1989; 12(2): 280-282, indexed in Pubmed: 2468137.

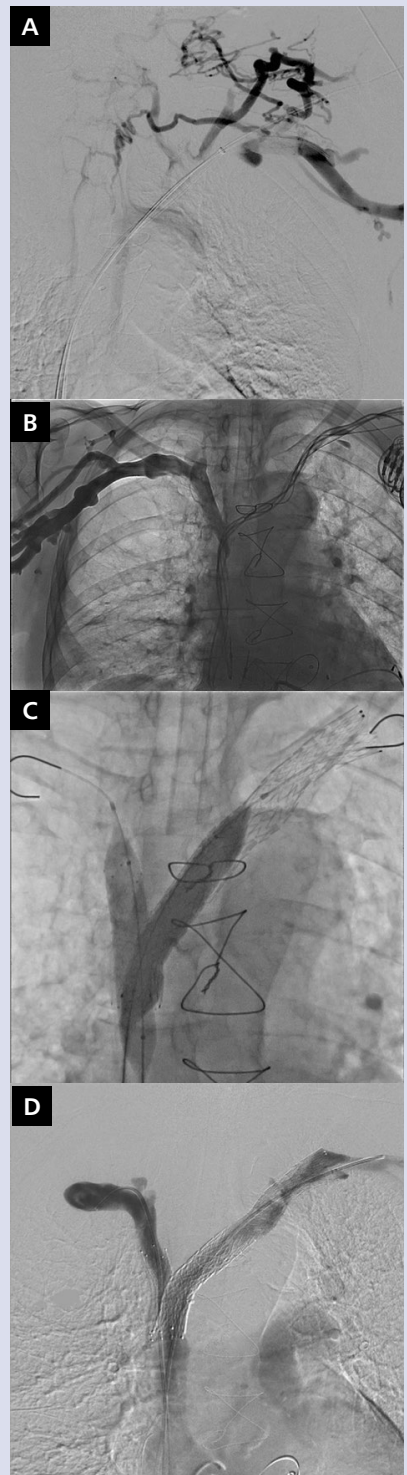

Figure 2. Left brachiocephalic vein (BCV) occlusion with collaterals (A) and critical stenosis of the right $\mathrm{BCV}$ adjacent to the superior vena cava (B); C. Stents post-dilatation - kissing-balloon technique; D. 24-month follow-up phlebography
Implantation of pacemakers, especially multi-lead systems, is performed increasingly often. Leads located inside the veins chronically irritate the vascular wall inducing an inflammatory response causing neointima overgrowth and thrombosis, and leading to vessel lumen narrowing or even occlusion. It is observed in $\sim 45 \%$ of cases but causes clinical symptoms of vascular occlusion, including superior vena cava syndrome (SVCS), in only $0.35 \%-1.5 \%$ [1-3]. We present two cases of endovascular treatment of severe, symptomatic venous occlusion in patients with an implantable cardiac pacemaker. A Venovo ${ }^{\circledR}$ (Bard, Covington, GA, USA) self-expanding stent, dedicated for the venous system, was used in both cases. The procedures were performed by a multidisciplinary team in the hybrid angiography suite, using general anaesthesia and with full cardioanaesthetic and cardiosurgical support. Case 1: A 52-year-old patient who underwent biological mitral valve replacement in 2015 had a dual-chamber pacemaker (DDD) implanted for treatment of third-degree atrioventricular block. One year later the patient experienced left hand oedema, worsening his quality of life. Critical stenosis of the left brachiocephalic vein (BCV) and collaterals were found during phlebography (Fig. 1A). The procedure was started with transvenous lead extraction (TLE). The stenoses were dilated with Atlas $^{\text {TM }}$ (Bard, Covington, GA, USA) $12 / 60 \mathrm{~mm}$ and 18/60 mm balloon catheters, and afterwards two overlapping Venovo $^{\circledast}$ stents were implanted: $20 / 80 \mathrm{~mm}$ and $16 / 80 \mathrm{~mm}$, covering the entire length of the stenosis. Two pacemaker leads were implanted through the stents providing DDD-stimulation (Fig. 1B). No periprocedural complications occurred. Case 2: A 65-year-old patient with ischaemic cardiomyopathy and a history of multiple procedures: coronary artery bypass grafting (2005), biological mitral valve replacement (2005), tricuspid valve angioplasty (2007), implantation of cardioverter-defibrillator (2010) and its replacement with a cardiac resynchronisation therapy device (2012), and implantation of an additional atrial lead (2015), presented with symptoms of severe SVCS, rapidly progressing from 2015. Phlebography revealed occlusion of the left BCV and collaterals and critical stenosis of the proximal part of the right BCV (Fig. 2A, B). TLE was perfomed, followed by angioplasty of both BCVs was using Atlas TM $12 / 60 \mathrm{~mm}$ and $18 / 60 \mathrm{~mm}$ balloon catheters. A Venovo ${ }^{\circledR} 16 / 140 \mathrm{~mm}$ stent was implanted into the left BCV and an Epic ${ }^{\circledast}$ (Boston Scientific, Marlborough, MA, USA) $14 \times 40 \mathrm{~mm}$ stent was placed in the right BCV. Kissing-balloon angioplasty of stents was performed (Fig. 2C). A subcutaneous defibrillation system was implanted. No complications were observed. In both cases, a vitamin $\mathrm{K}$ antagonist was initiated one day after the procedure. Both patients presented complete withdrawal of SVCS symptoms. No symptoms of restenosis were observed during 24-month follow-up (Figs. 1C, 2D). The unique structure of the stents prevents them from being crushed between the clavicle and the first rib. The physiology of the venous system is less understood than the arterial system and remains a challenge to physicians, who use central veins increasingly often, with little thought about late complications. Surgical treatment of central venous pathologies is very difficult and endovascular treatment has been less effective. In our opinion, efficient and durable treatment is possible thanks to new venous-designed stents. There is an urgent need to build multidisciplinary, experienced teams consisting of electrocardiologists, sonographers, interventional radiologists, angiologists, cardiosurgeons, and anaesthesiologists. All procedures must be performed in the hybrid angiography suite, using all-modality guidance (ultrasound, transoesophageal echocardiography, fluoroscopy) and cardiosurgical support.

Address for correspondence:

Dr. Radosław Pietura, Department of Radiography, Medical University of Lublin, ul. Staszica 11, 20-081 Lublin, Poland, e-mail: radoslawpietura@gmail.com

Conflict of interest: none declared

Kardiologia Polska Copyright (c) Polish Cardiac Society 2018 